\title{
A standardisation proof for algebraic pattern calculi
}

\author{
Delia Kesner \\ PPS, CNRS and Université Paris Diderot \\ France \\ Delia.Kesner@pps.jussieu.fr
}

\author{
Carlos Lombardi \\ Depto. de Ciencia y Tecnología \\ Univ. Nacional de Quilmes \\ Argentina \\ clombardi@unq.edu.ar
}

\author{
Alejandro Ríos \\ Depto. de Computación \\ Facultad de Cs. Exactas y Naturales \\ Univ. de Buenos Aires - Argentina \\ rios@dc.uba.ar
}

\begin{abstract}
This work gives some insights and results on standardisation for call-by-name pattern calculi. More precisely, we define standard reductions for a pattern calculus with constructor-based data terms and patterns. This notion is based on reduction steps that are needed to match an argument with respect to a given pattern. We prove the Standardisation Theorem by using the technique developed by Takahashi [14] and Crary [2] for $\lambda$-calculus. The proof is based on the fact that any development can be specified as a sequence of head steps followed by internal reductions, i.e. reductions in which no head steps are involved.
\end{abstract}

\section{Introduction}

Pattern Calculi: Several calculi, called pattern calculi, have been proposed in order to give a formal description of pattern matching; i.e. the ability to analyse the different possible forms of the argument of a function in order to decide among different alternative definition clauses.

The pattern matching operation is the kernel of the evaluation mechanism of all these formalisms, basically because reduction can only be fired when the argument passed to a given function matches its pattern specification. An analysis of various pattern calculi based on different notions of pattern matching operations and different sets of allowed patterns can be found in [8].

Standardisation: A fundamental result in the $\lambda$-calculus is the Standardisation Theorem, which states that if a term $M \beta$-reduces to a term $N$, then there is a standard $\beta$-reduction sequence from $M$ to $N$ which can be seen as a canonical way to reduce terms. This result has several applications, e.g. it is used to prove the non-existence of reduction between given terms. One of its main corollaries is the quasi-leftmost-reduction theorem, which in turn is used to prove the non-existence of a normal form for a given term.

A first study on standardisation for call-by-name $\lambda$-calculus appears in [3]. Subsequently, several standardisation methods have been devised, for example [1] Section 11.4, [14], [9] and [13].

While leftmost-outermost reduction gives a standard strategy for call-by-name $\lambda$-calculus, more refined notions of reductions are necessary to define standard strategies for call-by-value $\lambda$-calculus [13], first-order term rewriting systems [6, 15], Proof-Nets [4], etc.

All standard reduction strategies require the definition of some selected redex by means of a partial function from terms to redexes; they all give priority to the selected step, if possible. This selected redex is sometimes called external [11], but we will refer here to it as the head redex of a term.

It is also worth mentioning a generic standardisation proof [12] that can uniformly treat cal-by-name and call-by-value $\lambda$-calculus. It is parameterized over the set of values that allow to fire the beta-reduction rule. However, the set of values are defined there in a global sense, while in pattern calculi being a value strongly depends on the form of the given pattern.

Standardisation in Pattern Calculi: For call-by-name $\lambda$-calculus, any term of the form $(\lambda x . M) N$ is a redex, and the head redex for such a term is the whole term. In pattern calculi any term of the form

E. Bonelli (Ed.): 5th International Workshop on Higher-Order Rewriting (HOR'10)

EPTCS 49, 2011, pp. 58-72 doi 10.4204/EPTCS.49.5 (c) D.Kesner, C.Lombardi \& A.Ríos

This work is licensed under the Creative Commons Attribution-Share Alike License. 
$(\lambda p . M) N$ is a redex candidate, but not necessarily a redex. The parameter $p$ in such terms can be more complex than a single variable, and the whole term is not a redex if the argument $N$ does not match $p$, i.e., if $N$ does not verify the structural conditions imposed by $p$. In this case we will choose as head a reduction step lying inside $N$ (or even inside $p$ ) which makes $p$ and $N$ be closer to a possible match. While this situation bears some resemblance with call-by-value $\lambda$-calculus [13], there is an important difference: both the fact of $(\lambda p . M) N$ being a redex, and whether a redex inside $N$ could be useful to get $p$ and $N$ closer to a possible match, depend on both $N$ and $p$.

The aim of this contribution is to analyse the existence of a standardisation procedure for pattern calculi in a direct way, i.e. without using any complicated encoding of such calculi into some general computational framework [10]. This direct approach aims to put to evidence the fine interaction between reduction and pattern matching, and gives a standardisation algorithm which is specified in terms of the combination of computations of independent terms with partial computations of terms depending on some pattern. We hope to be able to extend this algorithmic approach to more sophisticated pattern calculi handling open and dynamic patterns [7].

The paper is organized as follows. Section 2 introduces the calculus, Section 3 gives the main concepts needed for the standardisation proof and the main results, Section 4 presents some lemmas used in the main proofs, Sections 5 and 6 show the main results used in the Standardisation Theorem proof and then the theorem itself; finally, Section 7 concludes and gives future research directions.

\section{The calculus}

We will study a very simple form of pattern calculus, consisting of the extension of standard $\lambda$-calculus with a set of constructors and allowing constructed patterns. This calculus appears for example in Section 4.1 in [8].

Definition 2.1 (Syntax) The calculus is built upon two different enumerable sets of symbols, the variables $x, y, z, w$ and the constants $c, a, b$; its syntactical categories are:

$$
\begin{aligned}
& \text { Terms } \quad M, N, Q, R \quad::=x|c| \lambda p \cdot M \mid M M \quad \text { DataTerms } \quad D \quad:=c \mid D M \\
& \text { Patterns } \quad p, q:=x \mid d \quad \text { DataPatterns } d::=c \mid d p
\end{aligned}
$$

Free and bound variables of terms are defined as expected as well as $\alpha$-conversion.

Definition 2.2 (Substitution) A susbsitution $\theta$ is a function from variables to terms with finite domain, where $\operatorname{dom}(\theta)=\{x: \theta(x) \neq x\}$. The extension of $\theta$ to terms is defined as expected. We denote $\theta::=\left\{x_{1} / M_{1}, \ldots, x_{n} / M_{n}\right\}$ wherever $\operatorname{dom}(\theta) \subseteq\left\{x_{1}, \ldots, x_{n}\right\}$. Moreover, for $\theta, v$ substitutions, $X$ a set of variables, we define

$$
\begin{aligned}
\operatorname{var}(\theta) & ::=\operatorname{dom}(\theta) \cup\left(\cup_{x \in \operatorname{dom}(\theta)} \mathrm{fv}(\theta x)\right) \\
v \theta & ::=\left(\cup_{x \in \operatorname{dom}(\theta)}\{x / \nu(\theta x)\}\right) \cup\left(\cup_{x \in(\operatorname{dom}(v)-\operatorname{dom}(\theta))}\{x / v x\}\right) \\
\left.\theta\right|_{X} & ::=\cup_{x \in X \cap \operatorname{dom}(\theta)}\{x / \theta x\}
\end{aligned}
$$

Definition 2.3 (Matching) Let $p$ be a pattern and $M$ a term which do not share common variables. Matching on $p$ and $M$ is a partial function yielding a substitution and defined by the following rules $(\uplus$ on substitutions denotes disjoint union with respect to their domains, being undefined if the domains have a non-empty intersection): 


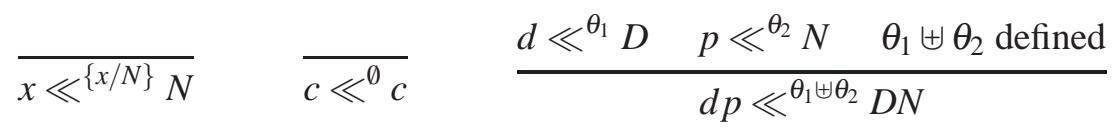

We write $p \ll M$ iff $\exists \theta p \ll^{\theta} M$. Remark that $p \ll M$ implies that $p$ is linear.

Definition 2.4 (Reduction step) We consider the following reduction steps modulo $\alpha$-conversion:

$$
\frac{M \rightarrow M^{\prime}}{M N \rightarrow M^{\prime} N} \text { SAppL } \frac{N \rightarrow N^{\prime}}{M N \rightarrow M N^{\prime}} \text { SAppR } \frac{p \ll^{\theta} N}{(\lambda p \cdot M) N \rightarrow \theta M} \text { SBeta } \frac{M \rightarrow M^{\prime}}{\lambda p \cdot M \rightarrow \lambda p \cdot M^{\prime}} \text { SAbs }
$$

By working modulo $\alpha$-conversion we can always assume in rule (SBeta) that $p$ and $N$ do not share common variables in order to compute matching.

\section{Lemma 2.5 (Basic facts about the calculus)}

a. (data pattern/term structure) Let $d \in$ DataPatterns (resp. $D \in$ DataTerms), then $d=c p_{1} \ldots p_{n}$ (resp. $D=c M_{1} \ldots M_{n}$ ) for some $n \geq 0$.

b. (data patterns only match data terms) Let $d \in$ DataPatterns, $M$ a term, such that $d \ll M$. Then $M \in$ DataTerms.

c. (minimal matches) If $p \ll^{\theta} M$ then $\operatorname{dom}(\theta)=\mathrm{fv}(p)$.

d. (uniqueness of match) If $p \ll^{\theta_{1}} M$ and $p \ll^{\theta_{2}} M$, then $\theta_{1}=\theta_{2}$.

Crucial to the standardisation proof is the concept of development, we formalize it through the relation $\triangleright$, meaning $M \triangleright N$ iff there is a development (not necessarily complete) with source $M$ and target $N$.

Definition 2.6 (Term and substitution development) We define the relation $\triangleright$ on terms and a corresponding relation $>$ on substitutions. The relation $\triangleright$ is defined by the following rules:

$$
\begin{aligned}
\frac{M \triangleright M}{M \triangleright R e f l} & \frac{M \triangleright M^{\prime}}{\lambda p \cdot M \triangleright \lambda p \cdot M^{\prime}} \text { DAbs } \\
\frac{M \triangleright M^{\prime} N \triangleright N^{\prime}}{M N \triangleright M^{\prime} N^{\prime}} \text { DApp } & \frac{M \triangleright M^{\prime} \quad \theta \triangleright \theta^{\prime} \quad p \ll \ll^{\theta} N}{(\lambda p \cdot M) N \triangleright \theta^{\prime} M^{\prime}} \text { DBeta }
\end{aligned}
$$

and $>$ is defined as follows: $\theta \triangleright \theta^{\prime}$ iff $\operatorname{dom}(\theta)=\operatorname{dom}\left(\theta^{\prime}\right)$ and $\forall x \in \operatorname{dom}(\theta) . \theta x \triangleright \theta^{\prime} x$

\subsection{Head step}

The definition of head step will take into account the terms $(\lambda p . M) N$ even if $p \nless N$. In such cases, the head redex will be inside $N$ as the patterns in this calculus are always normal forms (this will not be the case for more complex pattern calculi).

The selection of the head redex inside $N$ depends on both $N$ and $p$. This differs from standard call-by-value $\lambda$-calculus, where the selection depends only on $N$.

We show this phenomenon with a simple example. Let $a, b, c$ be constants and $N=\left(a R_{1}\right) R_{2}$, where $R_{1}$ and $R_{2}$ are redexes. The redexes in $N$ needed to achieve a match with a certain pattern $p$, and thus the selection of the head redex, depend on the pattern $p$.

Take for example different patterns $p_{1}=(a x)(b y), p_{2}=(a b x) y, p_{3}=(a b x)(c y), p_{4}=(a x) y$, and consider the term $Q=(\lambda p . M) N$. If $p=p_{1}$, then it is not necessary to reduce $R_{1}$ (because it already matches 
$x$ ) but it is necessary to reduce $R_{2}$, because no redex can match the pattern by; hence $R_{2}$ will be the head redex in this case. Analogously, for $p_{2}$ it is necessary to reduce $R_{1}$ but not $R_{2}$, for $p_{3}$ both are needed (in this case we will choose the leftmost one) and $p_{4}$ does match $N$, hence the whole $Q$ is the head redex. This observation motivates the following definition.

Definition 2.7 (Head step) The relations $\underset{h}{\rightarrow}$ (head step) and $\underset{p}{\rightsquigarrow}$ (preferred needed step to match pattern p) are defined as follows:

$$
\begin{aligned}
& \frac{M \underset{h}{\rightarrow} M^{\prime}}{M N \underset{h}{\rightarrow} M^{\prime} N} \text { HApp1 } \frac{p \ll{ }^{\theta} N}{(\lambda p \cdot M) N \underset{h}{\rightarrow} \theta M} \text { HBeta } \frac{N \rightsquigarrow N^{\prime}}{(\lambda p \cdot M) N \underset{h}{\rightarrow}(\lambda p \cdot M) N^{\prime}} \text { HPat } \\
& \frac{M \underset{h}{\rightarrow} M^{\prime}}{M \underset{d}{\rightsquigarrow} M^{\prime}} \text { PatHead } \quad \frac{D \underset{d}{\rightsquigarrow} D^{\prime}}{D M \underset{d p}{\rightsquigarrow} D^{\prime} M} \text { Pat1 } \quad \frac{M \rightsquigarrow M^{\prime} d \ll D}{D M \underset{d p}{\rightsquigarrow} D M^{\prime}} \text { Pat2 }
\end{aligned}
$$

The rule PatHead is intended for data patterns only, not being valid for variable patterns; we point this by writing a $d$ (data pattern) instead of a $p$ (any pattern) in the arrow subscript inside the conclusion.

We observe that the rule analogous to HPat in the presentation of standard reduction sequences for call-by-value $\lambda$-calculus in both [13] and [2] reads

$$
\frac{N \underset{h}{\rightarrow} N^{\prime}}{(\lambda p \cdot M) N \underset{h}{\rightarrow}(\lambda p \cdot M) N^{\prime}}
$$

reflecting the $N$-only-dependency feature aforementioned.

We see also that a head step in a term like $(\lambda p . M) N$ determined by rule HPat will lie inside $N$, but the same step will not necessarily be considered head if we analyse $N$ alone.

It is easy to check that if $M \rightsquigarrow M^{\prime}$ then $p \nless M$, avoiding any overlap between HBeta and HPat and also between Pat1 and Pat2. This in turn implies that all terms have at most one head redex. We remark also that the head step depends not only on the pattern structure but also on the match or lack of match between pattern and argument.

\section{Lemma 2.8 (Basic facts about head steps)}

a. (head reduction only if abstraction in head) Let $M$ be a term such that $M_{h} \rightarrow M^{\prime}$ for some $M^{\prime}$. Then $M=\left(\lambda p . M_{01}\right) M_{1} \ldots M_{n}$ with $n \geq 1$.

b. (head reduction only if no match) Let $M$ be a term such that $M \underset{h}{\rightarrow} M^{\prime}$ for some $M^{\prime}, d \in$ DataPatterns. Then $d \nless M$.

c. $\underset{p}{\rightsquigarrow}$ only if $\underset{h}{\rightarrow}$ or data term) Let $p$ be a pattern and let $M$ be a term such that $M \underset{p}{\rightsquigarrow} M^{\prime}$ for some $M^{\prime}$. Then either $M \in$ DataTerms or $M \underset{h}{\rightarrow} M^{\prime}$.

Proof Item (a) is trivial. Item (b) uses Item (a) and L. 2.5 (b). Item (ㄷ) is trival by definition of $\underset{p}{m}$. 


\section{Main concepts and ideas needed for the standardisation proof}

In order to build a standardisation proof for constructor based pattern calculi we chose to adapt the one in [14] for the call-by-name $\lambda$-calculus, later adapted to call-by-value $\lambda$-calculus in [2], over the classical presentation of [13].

The proof method relies on a h-development property stating that any development can be split into a leading sequence of head steps followed by a development in which no head steps are performed; this is our Corollary 5.4 which corresponds to the so-called "main lemma" in the presentations by Takahashi and Crary.

Even for a simple form of pattern calculus such as the one presented in this contribution, both the definitions (as we already mentioned when defining head steps) and the proofs are non-trivial extensions of the corresponding ones for standard $\lambda$-calculus, even in the framework of call-by-value. As mentioned before, the reason is the need to take into account, for terms involving the application of a function to an argument, the pattern of the function parameter when deciding whether a redex inside the argument should be considered as a head redex.

In order to formalize the notion of "development without occurrences of head steps", an internal development relation will be defined. The dependency on both $N$ and $p$ when analysing the reduction steps from a term like $(\lambda p . M) N$ is shown in the rule IApp2.

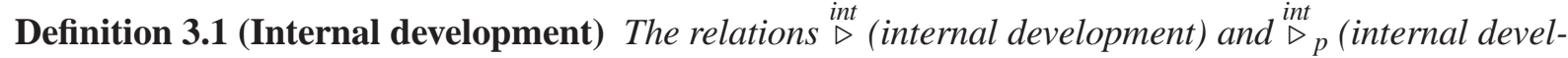
opment with respect to the pattern $p$ ) are defined as follows:

$$
\begin{aligned}
& \underset{M \stackrel{\text { int }}{\triangleright} M}{\operatorname{IRefl}} \frac{M \triangleright M^{\prime}}{\lambda p \cdot M \stackrel{\text { int }}{\triangleright} \lambda p \cdot M^{\prime}} \text { IAbs } \quad \frac{M \neq \lambda p . M_{1} \quad M \stackrel{\text { int }}{\triangleright} M^{\prime} N \triangleright N^{\prime}}{M N \stackrel{i n t}{\triangleright} M^{\prime} N^{\prime}} \text { IApp1 } \\
& \frac{M \triangleright M^{\prime} \quad N \stackrel{\text { int }}{p}_{p} N^{\prime}}{(\lambda p . M) N \triangleright \text { int }\left(\lambda p \cdot M^{\prime}\right) N^{\prime}} \text { App2 } \quad \frac{N \triangleright N^{\prime} p \ll N}{N{ }^{i n t}{ }_{p} N^{\prime}} \text { PMatch } \quad \frac{N{ }^{i n t} N^{\prime}}{N \triangleright_{c} N^{\prime}} \text { PConst }
\end{aligned}
$$

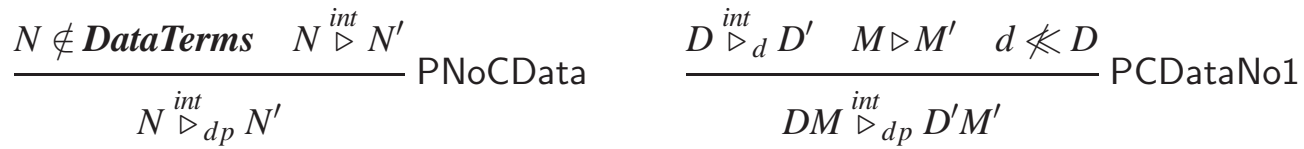

$$
\begin{aligned}
& \frac{D \triangleright D^{\prime} \quad M \stackrel{i n t}{\triangleright}_{p} M^{\prime} \quad d \ll D \quad p \ll M}{D M \stackrel{i n t}{\triangleright}_{d p} D^{\prime} M^{\prime}} \text { PCDataNo2 } \\
& \frac{D \triangleright D^{\prime} \quad M \triangleright M^{\prime} \quad d \ll D \quad p \ll M \quad d p \ll D M}{D M \stackrel{i n t}{\triangleright}_{d p} D^{\prime} M^{\prime}} \text { PCDataNo3 }
\end{aligned}
$$

Remark that rule PCDataNo3 is useful to deal with non-linear patterns.

Thus for example, $a b((\lambda y \cdot y) c) \stackrel{\text { int }}{\triangleright \text { axx }} a b c$ since $a b \triangleright a b,(\lambda y \cdot y) c \triangleright c, a x \ll a b, x \ll(\lambda y \cdot y) c$ but $a x x \ll$ $a b((\lambda y \cdot y) c)$.

We observe also that if $N \stackrel{\text { int }}{\triangleright} N^{\prime}$ or $N \stackrel{i n t}{\triangleright}_{p} N^{\prime}$ then $N \triangleright N^{\prime}$.

The following lemma analyses data / non-data preservation 


\section{Lemma 3.2 (Development and data)}

a. (internal development cannot create data terms) Let $M \notin$ DataTerms, $N$ such that $M \stackrel{\text { int }}{\triangleright} N$. Then $N \notin$ DataTerms

b. (development from data produces always data) Let $M \in$ DataTerms, $N$ such that $M \triangleright N$. Then $N \in$ DataTerms

The formal description of the h-development condition takes a form of an additional binary relation. This relation corresponds to the one called strong parallel reduction in [2].

Definition 3.3 (H-development) We define the relations $\underset{h}{\triangleright}$ and $\vec{h}_{h}$. Let $M, N$ be terms; $v, \theta$ substitutions.

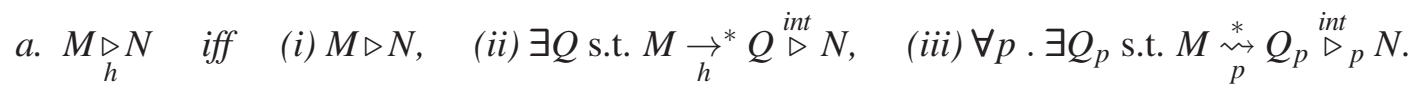

b. $v \underset{h}{ } \theta \quad$ iff $\quad$ (i) $\operatorname{Dom}(v)=\operatorname{Dom}(\theta), \quad$ (ii) $\forall x \in \operatorname{Dom}(v) \cdot \underset{h}{v x} \boldsymbol{\theta} \theta$.

The clause (iii) in the definition of $\triangleright$ shows the dependency on the patterns that was already noted in the definitions of head step and internal development.

This clause is needed when proving that all developments are h-developments; let's grasp the reason through a brief argument. Suppose we want to prove that a development inside $N$ in a term like $(\lambda p . M) N$ is an h-development. The rules to be used in this case are HPat (Def.2.7) and IApp2 (Def. 3.1). Therefore we need to perform an analysis relative to the pattern p; and this is exactly expressed by clause (iii). Consequently the proof of clause (ii) for a term needs to consider clause (iii) (instantiated to a certain pattern) for a subterm; this is achieved by including clause (iii) in the definition and by performing an inductive reasoning on terms.

\section{Auxiliary results}

We collect in this section some results needed to complete the main proofs in this article.

Lemma 4.1 (pattern-head reduction only if there is no match)

Let $M, N$ be terms, $p$ a pattern, such that $\underset{p}{\longrightarrow} N$. Then $p \nless M$.

Proof Using L. 2.8(b).

\section{Lemma 4.2 (development cannot lose matches)}

Let $M, N$ be terms, $p$ a pattern, such that $M \triangleright N$ and $p \ll^{v} M$. Then $p \ll^{\theta} N$ for some $\theta$ such that $v \triangleright \theta$.

Proof Induction on $p \ll^{v} M$. The axioms can be checked trivially. For the rule, let $M=M_{1} M_{2}, N=$ $N_{1} N_{2}, p=p_{1} p_{2}$ and $v=v_{1} \uplus v_{2} ; p$ is linear since it matches a term. The only rules applicable for $M \triangleright N$ are DRefl or DApp; DBeta is not applicable because $M_{1} \in$ DataTerms. If DRefl was used, the lemma holds trivially taking $\theta=v$. If DApp was used, we apply the IH on both hypotheses obtaining $p_{i} \ll^{\theta_{i}} N_{i}$ with $v_{i}>\theta_{i}$; by L. 2.5. (ㄷ) and the linearity of $p$ we know $\theta=\theta_{1} \uplus \theta_{2}$ is well-defined; it is easy to check that $\theta$ satisfies the lemma conditions.

Lemma $4.3 \stackrel{\text { int }}{\triangleright}$ cannot create match $)$

Let $M, N$ be terms, $p$ a pattern, such that $M \stackrel{\text { int }}{\triangleright}$ p $N$. Then $p \nless M$ implies $p \nless N$. 
Proof Induction on $M \stackrel{i n t}{\triangleright}{ }_{p} N$ by rule analysis

PMatch not applicable as $p \nless M$.

PConst in this case the condition $p \nless M$ implies $p \nless N$ equates to $M \neq p$ implies $N \neq p$, as $p$ is a constant.

The rule premise reads $M \stackrel{i n t}{\triangleright} N$ : if rule IRefl was used then $N \neq p$ by hypothesis, else the $\stackrel{i n t}{\triangleright}$ rule conclusions exclude the possibility of $N$ being a constant.

PNoCData $M \notin$ DataTerms and $M \stackrel{\text { int }}{\triangleright} N$ by rule hyp., then $N \notin$ DataTerms by L. 3.2. (a), finally $p \nless N$ by L. 2.5. (b).

PCDataNo1 By the IH, as rule hyp. includes both $D{\stackrel{i n t}{\triangleright_{d}}}_{d} D^{\prime}$ and $d \nless D$ being $M=D T$ and $p=d p^{\prime}$.

PCDataNo2 Similar to the former considering $p=d p^{\prime}$ and using $T \stackrel{\text { int }}{\triangleright_{p^{\prime}}} T^{\prime}$ and $p^{\prime} \nless T$.

PCDataNo3 In this case $M=D M^{\prime}, p=d p^{\prime}, d \ll^{\theta} D, p^{\prime} \ll^{\theta^{\prime}} M^{\prime}$ and $d p^{\prime} \ll D M^{\prime}$. We necessarily have that $\theta \uplus \theta^{\prime}$ is not defined hence $p$ is not linear so that $p \ll N$ also holds.

\section{Lemma 4.4 (left-pattern-head implies whole-pattern-head)}

Let $p_{1}, p_{2}$ be patterns and $M_{1}, N_{1}, M_{2}$ be terms such that $M_{1} \underset{p_{1}}{\rightsquigarrow} N_{1}$. Then $M_{1} M_{2} \underset{p_{1} p_{2}}{\rightsquigarrow} N_{1} M_{2}$.

Proof It is clear that $p_{1} \notin \operatorname{Var}$, because there is no $N_{1}$ such that $M_{1} \rightsquigarrow N_{1}$ if $x \in \operatorname{Var}$.

If PatHead applied in $M_{1} \underset{p_{1}}{\rightsquigarrow} N_{1}$, then $M_{1} \underset{h}{\rightarrow} N_{1}$, by HApp1 $M_{1} M_{2} \underset{h}{\rightarrow} N_{1} M_{2}$, and finally by PatHead $M_{1} M_{2} \underset{p_{1} p_{2}}{\rightsquigarrow} N_{1} M_{2}$.

If either Pat1 or Pat2 applied in $M_{1} \underset{p_{1}}{\rightsquigarrow} N_{1}$, then $M_{1}$ is clearly a data term, Then $M_{1} M_{2} \underset{p_{1} p_{2}}{\rightsquigarrow} N_{1} M_{2}$ by Pat1.

\section{Lemma 4.5 (matching is compatible with substitution)}

Let $M$ be a term, $p$ a pattern and $\theta$ a substitution such that $p \ll^{\theta} M$. Then for any substitution $v$, the following holds: $p \ll^{\gamma} v M$ where $\gamma=\left.v \theta\right|_{\mathrm{fv}(p)}$.

Proof By induction on the match. The axioms can be checked trivially given L. 2.5. (C).

We analyze the rule applied in this context

$$
\frac{d \ll^{\theta_{1}} M_{1} \quad p^{\prime} \ll^{\theta_{2}} M_{2}}{d p^{\prime}=p \ll{ }^{\theta=\theta_{1} \uplus \theta_{2}} M=M_{1} M_{2}}
$$

Applying the IH on both hypotheses and then using the rule gives $d p^{\prime} \ll^{\left.\left.\left(v \theta_{1}\right)\right|_{\mathrm{fv}(d)} \uplus\left(v \theta_{2}\right)\right|_{\mathrm{fv}(p)}} M_{1} M_{2}$; an easy check of $\left.\left.\left(v \theta_{1}\right)\right|_{f \mathrm{v}(d)} \uplus\left(v \theta_{2}\right)\right|_{\mathrm{fv}\left(p^{\prime}\right)}=\left.\left(v\left(\theta_{1} \uplus \theta_{2}\right)\right)\right|_{f \mathrm{v}\left(d p^{\prime}\right)}$ concludes the proof.

Lemma 4.6 (development is compatible with substitution)

Let $M, N$ be terms and $v, \theta$ substitutions, such that $M \triangleright N$ and $v \triangleright \theta$. Then $v M \triangleright \theta N$

Proof By induction on $M \triangleright N$ by rule analysis.

For DRefl the thesis amounts to $v M \triangleright \theta M$, which can be checked by a simple induction on $M$. DAbs and DApp can be simply verified by the IH. 
For DBeta first we mention a technical result which will be used. Let $\theta, \tau$ be substitutions such that $\operatorname{dom}(\tau) \cap \operatorname{var}(\theta)=\emptyset$, then

$$
\left(\left.(\theta \tau)\right|_{\operatorname{dom}(\tau)}\right) \theta=\theta \tau
$$

this can be easily checked comparing the effect of applying both substitutions to an arbitrary variable.

Let's analyze the rule premises and conclusion applied in this context

$$
\frac{M_{1} \triangleright M_{1}^{\prime} \quad \tau \triangleright \tau^{\prime} \quad p \ll^{\tau} M_{2}}{M=\left(\lambda p . M_{1}\right) M_{2} \triangleright \tau^{\prime} M_{1}^{\prime}=N}
$$

As we can freely choose the variables appearing in $p$, we assume $f \mathrm{v}(p) \cap(\operatorname{var}(v) \cup \operatorname{var}(\theta))=\emptyset$. By L. 2.5. (C) we know $\operatorname{dom}(\tau)=\operatorname{dom}\left(\tau^{\prime}\right)=\mathrm{fv}(p)$.

We apply the IH on $M_{1} \triangleright M_{1}^{\prime}$ and also on $\tau x \triangleright \tau^{\prime} x$ for each $x \in \operatorname{dom}(\tau)$ to conclude $v M_{1} \triangleright \theta M_{1}^{\prime}$ and $\left.\left.(\nu \tau)\right|_{\operatorname{dom}(\tau)} \triangleright\left(\theta \tau^{\prime}\right)\right|_{\operatorname{dom}(\tau)}$ respectively. Furthermore, from $p \ll^{\tau} M_{2}$ and L. 4.5 we conclude $p \ll^{\left(v \tau \tau_{\operatorname{dom}(\tau)}\right)}$ $v M_{2}$.

We use DBeta from the three conclusions above to obtain

$$
v M=\left(\lambda p . v M_{1}\right)\left(v M_{2}\right) \triangleright\left(\left.\left(\theta \tau^{\prime}\right)\right|_{\operatorname{dom}(\tau)}\right)\left(\theta M_{1}^{\prime}\right)
$$

To check $\theta N=\theta\left(\tau^{\prime} M_{1}^{\prime}\right)=\left(\left.\left(\theta \tau^{\prime}\right)\right|_{\operatorname{dom}(\tau)}\right)\left(\theta M_{1}^{\prime}\right)$ it is enough to verify $\theta \tau^{\prime}=\left(\left.\left(\theta \tau^{\prime}\right)\right|_{\operatorname{dom}(\tau)}\right) \theta$, the latter can be easily checked by (1).

\section{Lemma 4.7 (head reduction is compatible with substitution)}

(i) Let $M, N$ be terms and $v$ a substitution such that $M \underset{h}{\rightarrow} N$. Then $v M \underset{h}{\rightarrow} v N$.

(ii) Let $M, N$ be terms, $p$ a pattern and $v$ a substitution such that $M \underset{p}{\rightsquigarrow} N$. Then $v M \underset{p}{v} v N$.

Proof (sketch)

Both items are proved by simultaneous induction on $M \underset{h}{\rightarrow} N$ and $M \underset{p}{M} N$.

We use L. 4.5 for case HBeta, the IH and L. 4.5 for case Pat2, and just the IH for the remaining cases.

\section{H-developments}

The aim of this section is to prove that all developments are h-developments.

We found easier to prove separately that the h-development condition is compatible with the language constructs, diverging from the structure of the proofs in [2].

\section{Lemma 5.1 ( $\triangleright$ is compatible with abstraction)}

Let $M, N$ be terms such that $\underset{h}{\triangleright} N$. Then $\lambda q . M \underset{h}{\triangleright} \lambda q . N$ for any pattern $q$.

Proof Part (i) trivially holds by hyp. (i) and DAbs.

Part (ii): by hyp. (i) and IAbs we get $\lambda q . M \stackrel{i n t}{\triangleright} \lambda q . N$. Then $Q=\lambda q \cdot M$.

Part (iii): if $p \in \operatorname{Var}$ then PMatch applies, if $p$ is a constant or a compound data pattern then PConst or PNoCData apply respectively as $(\lambda q \cdot M) \stackrel{i n t}{\triangleright}(\lambda q . N)$. In all cases we obtain $(\lambda q \cdot M) \stackrel{i n t}{\triangleright}{ }_{p}(\lambda q . N)$. Then $Q=\lambda q \cdot M$. 


\section{Lemma 5.2 ( $\triangleright$ is compatible with application)}

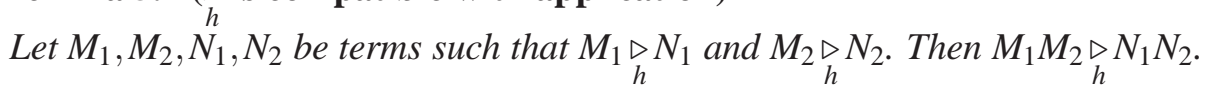

Proof Part $(i)$ is immediate by the hypotheses $(i)$ and DApp.

Let's prove part (ii).

We first use hypothesis (ii) on $M_{1} \triangleright{ }_{h} N_{1}$ to obtain $M_{1} \vec{h}^{*} Q_{1} \stackrel{i n t}{\triangleright} N_{1}$ and subsequently apply HApp1 to $M_{1} \underset{h}{*} Q_{1}$ to get

$$
M_{1} M_{2} \vec{h}^{*} Q_{1} M_{2}
$$

Either $Q_{1}$ is an abstraction or not.

Assume $Q_{1}$ is not an abstraction. Since $Q_{1} \stackrel{i n t}{\triangleright} N_{1}$ and $M_{2} \triangleright N_{2}$, we apply IApp1 so that $Q_{1} M_{2} \triangleright{ }^{i n t} N_{1} N_{2}$; this together with (2) gives the desired result.

Now assume $Q_{1}=\lambda p \cdot Q_{12}$. We use the hyp. (iii) on $\underset{h}{M_{2}} \underset{h}{\triangleright} N_{2}$, obtaining $\underset{p}{M_{2}} \underset{p}{\rightsquigarrow^{*}} Q_{2} \stackrel{i n t}{\triangleright}{ }_{p} N_{2}$ and then we apply HPat to get

$$
Q_{1} M_{2} \underset{h}{\rightarrow^{*}} Q_{1} Q_{2}
$$

Moreover, as $Q_{1}=\lambda p \cdot Q_{12} \stackrel{i n t}{\triangleright} N_{1}$, the only applicable rules are IRefl or IAbs, and in both cases $N_{1}=$ $\lambda$ p. $N_{12}$ and $Q_{12} \triangleright N_{12}$.

We now use IApp2 with premises $Q_{12} \triangleright N_{12}$ and $Q_{2} \stackrel{i n t}{p}_{p} N_{2}$ to get

$$
Q_{1} Q_{2}=\left(\lambda p \cdot Q_{12}\right) Q_{2} \stackrel{\text { int }}{\triangleright}\left(\lambda p \cdot N_{12}\right) N_{2}=N_{1} N_{2}
$$

The desired result is obtained by (2), (3) and (4).

Let's prove part (iii).

If $p \in \operatorname{Var}$ we are done by $(i)$ and PMatch; we thus get $M_{1} M_{2} \stackrel{i n t}{\triangleright}{ }_{p} N_{1} N_{2}$ so that $Q=M_{1} M_{2}$.

If $p=c$ then using (ii) we obtain $M_{1} M_{2} \vec{h}^{*} Q \stackrel{i n t}{\triangleright} N_{1} N_{2}$ for some $Q$; we apply PatHead and PConst to get $M_{1} M_{2} \underset{c}{\stackrel{*}{\rightarrow}} Q$ and $Q \stackrel{i n t}{\triangleright}{ }_{c} N_{1} N_{2}$ respectively, concluding the proof for this case.

Consider $p=p_{1} p_{2}$ with $p_{1}$ a data pattern and $p_{2}$ a pattern.

We use the hyp. (iii) on $M_{1} \underset{h}{\triangleright} N_{1}$, getting $M_{1} \underset{p_{1}}{\stackrel{*}{*}} Q_{1} \stackrel{i n t}{\triangleright_{p_{1}}} N_{1}$. Let us define $R_{1}$ as follows: if there is a data term in the sequence $M_{1} \underset{p_{1}}{\stackrel{*}{*}} Q_{1}$ then $R_{1}$ is the first of such terms; otherwise $R_{1}$ is $Q_{1}$. In both cases $M_{1} \underset{p_{1}}{\stackrel{*}{\rightsquigarrow}} R_{1} \underset{p_{1}}{\stackrel{*}{*}} Q_{1}$. We necessarily have $M_{1} \vec{h}^{*} R_{1}$ by PatHead, then $M_{1} M_{2} \vec{h}^{*} R_{1} M_{2}$ by HApp1 and subsequently $M_{1} M_{2} \underset{p}{\rightsquigarrow} R_{1} M_{2}$ by PatHead.

We conclude $M_{1} M_{2} \underset{p}{\stackrel{*}{\sim}} Q_{1} M_{2}$, trivially if $Q_{1}=R_{1}$, and applying Pat1 to $R_{1} \underset{p_{1}}{\stackrel{*}{*}} Q_{1}$ to obtain $R_{1} M_{2} \underset{p}{\stackrel{*}{*}}$ $Q_{1} M_{2}$ otherwise. 
If $Q_{1}=\left(\lambda q \cdot Q_{1}^{\prime}\right)$ then we use the hyp. (iii) on $M_{2} \triangleright{ }_{h} N_{2}$ getting $M_{2} \underset{q}{\stackrel{*}{\gtrless}} Q_{2} \stackrel{i n t}{\triangleright}{ }_{q} N_{2}$.

We apply HPat to $M_{2} \underset{q}{\mathfrak{w}^{*}} Q_{2}$ getting $Q_{1} M_{2} \vec{h}^{*} Q_{1} Q_{2}$; therefore we obtain $Q_{1} M_{2} \underset{p}{\stackrel{*}{*}} Q_{1} Q_{2}$ by PatHead.

In the other side $Q_{1}=\left(\lambda q \cdot Q_{1}^{\prime}\right) \triangleright N_{1}$, therefore $N_{1}=\left(\lambda q \cdot N_{1}^{\prime}\right)$ and $Q_{1}^{\prime} \triangleright N_{1}^{\prime}$.

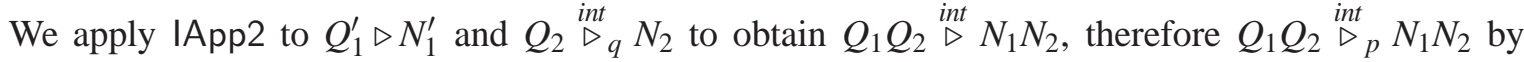
PNoCData. We thus get the desired result taking $Q_{p}=Q_{1} Q_{2}$.

If $Q_{1}$ is not an abstraction and $Q_{1} \notin$ DataTerms, then only PConst or PNoCData can justify $Q_{1} \stackrel{\text { int }}{\triangleright p_{1}}$ $N_{1}$, thus implying $Q_{1} \stackrel{i n t}{\triangleright} N_{1}$; this together with the hypothesis (i) $M_{2} \triangleright N_{2}$ gives $Q_{1} M_{2} \stackrel{i n t}{\triangleright} N_{1} N_{2}$ by IApp1, hence $Q_{1} M_{2} \stackrel{i n t}{\triangleright}_{p} N_{1} N_{2}$ by PNoCData. We get the desired result by taking $Q_{p}=Q_{1} M_{2}$.

If $Q_{1} \in$ DataTerms we anaylise the different alternatives for the matching between $p_{1} p_{2}$ and $Q_{1} M_{2}$.

Assume $p_{1} \nless Q_{1}$. In this case we apply PCDataNo1 to $Q_{1} \stackrel{i n t}{\triangleright_{p_{1}}} N_{1}$ and $M_{2} \triangleright N_{2}$ to obtain $Q_{1} M_{2} \triangleright_{p}$ $N_{1} N_{2}$ and thus the desired result holds by taking $Q_{p}=Q_{1} M_{2}$.

Assume $p_{1} \ll Q_{1}$ and $p_{2} \nless M_{2}$. In this case we use the hyp. (iii) on $M_{2} \underset{h}{\triangleright} N_{2}$ to get $\underset{M_{2}}{\stackrel{*}{\sim}} \underset{p_{2}}{\stackrel{i n t}{\triangleright}} Q_{p_{2}} N_{2}$, then apply Pat2 to get $Q_{1} M_{2} \underset{p}{\stackrel{*}{\sim}} Q_{1} Q_{2}$. Finally from $Q_{1} \stackrel{i n t}{\triangleright p_{1}} N_{1}$ and $Q_{2} \stackrel{i n t}{\triangleright}_{p_{2}} N_{2}$ we obtain $Q_{1} Q_{2} \stackrel{i n t}{\triangleright}_{p} N_{1} N_{2}$ by either PCDataNo2, PCDataNo3 or PMatch. We get the desired result by taking $Q_{p}=Q_{1} Q_{2}$.

Finally assume $p_{1} \ll Q_{1}$ and $p_{2} \ll Q_{2}$. In this case the hypotheses imply in particular $Q_{1} \triangleright N_{1}$ and $M_{2} \triangleright N_{2}$. We thus conclude $Q_{1} M_{2} \triangleright_{p}{ }_{p} N_{1} N_{2}$ using either PMatch or PCDataNo3 (depending on whether $p \ll Q_{1} M_{2}$ or not), getting the desired result by taking $Q_{p}=Q_{1} M_{2}$.

Now we proceed with the proof of the h-development property. The generalization of the statement involving $\underset{h}{ }$ is needed to conclude the proofl , as can be seen in the DBeta case below.

\section{Lemma 5.3 (Generalized h-developments property)}

Let $M, N$ be terms and $v, \theta$ substitutions, such that $M \triangleright N$ and $v \nabla_{h} \theta$.

Then $v M \underset{h}{\triangleright} \theta N$

Proof By induction on $M \triangleright N$ analyzing the rule used in the last step of the derivation.

DRefl in this case $N=M$, we proceed by induction on $M$

- $M=x \in \operatorname{Dom}(v)$, in this case $v M=v x \underset{h}{\triangleright} \theta x=\theta N$ by hypothesis.

- $M=x \notin \operatorname{Dom}(v)$, in this case $v M=x \underset{h}{\triangleright} x=\theta N$.

- $M=M_{1} M_{2}$, in this case $v M_{1} \underset{h}{\triangleright} \theta M_{1}$ and $v M_{2} \underset{h}{\triangleright} \theta M_{2}$ hold by the IH. The desired result is obtained by L. 5.2

- $M=\lambda p \cdot M_{1}$, in this case $v M_{1} \underset{h}{\triangleright} \theta M_{1}$ holds by the IH. The desired result is obtained by L. 5.1

\footnotetext{
${ }^{1}$ In [2] the compatibility of h-development with substitutions is stated as a separate lemma; for pattern calculi we could not find a proof of compatibility with substitution independent of the main h-development result.
} 
DAbs in this case $M=\lambda p \cdot M_{1}, N=\lambda p \cdot N_{1}, M_{1} \triangleright N_{1}$.

Using the IH on $M_{1} \triangleright N_{1}$ we obtain $v M_{1} \triangleright \underset{h}{\theta} \theta N_{1}$, the desired result is obtained by L. 5.1 ,

DApp in this case $M=M_{1} M_{2}, N=N_{1} N_{2}, M_{i} \triangleright N_{i}$.

Using the IH on both rule premises we obtain $v M_{i} \triangleright \theta N_{i}$, the desired result is obtained by L.5.2

DBeta Let's write down the rule instantiation

$\frac{M_{12} \triangleright N_{12} \quad \tau \triangleright \tau^{\prime} \quad q \ll{ }^{\tau} M_{2}}{M=\left(\lambda q \cdot M_{12}\right) M_{2} \triangleright \tau^{\prime} N_{12}=N}$

(i) can be obtained by hypotheses $M \triangleright N$ and $v \nabla_{h} \theta$, and then L.4.6,

For [ ( iii) if $p \in \operatorname{Var}]$ we are done by $(i)$ and PMatch.

For [ (iii) if $p=d$ ] and also for (ii): we know both $M \underset{h}{\rightarrow} \tau M_{12}$ and $\underset{p}{M} \tau M_{12}$, then by L.4.7

$$
v M \underset{h}{\rightarrow} v\left(\tau M_{12}\right) \quad \text { and } \quad v M \underset{p}{\rightsquigarrow} v\left(\tau M_{12}\right)
$$

We apply the IH on each $\tau x \triangleright \tau^{\prime} x$, obtaining $(v \tau) x=v(\tau x) \underset{h}{\triangleright} \theta\left(\tau^{\prime} x\right)=\left(\theta \tau^{\prime}\right) x$ for all $x \in \operatorname{Dom}(\tau)$. Moreover, if $x \in \operatorname{Dom}(v)-\operatorname{Dom}(\tau)$ then $(v \tau) x=v x \underset{h}{\triangleright} \theta x=\left(\theta \tau^{\prime}\right) x$ by hypothesis.

Consequently, $v \tau \underset{h}{\nabla} \theta \tau^{\prime}$. Now we use the IH on $M_{12} \triangleright N_{12}$ taking $v \tau \underset{h}{\triangleright_{h}} \theta \tau^{\prime}$ as second hypothesis to obtain

$$
v\left(\tau M_{12}\right)=(v \tau) M_{12} \underset{h}{\triangleright}\left(\theta \tau^{\prime}\right) N_{12}=\theta\left(\tau^{\prime} N_{12}\right)=\theta N
$$

This result along with (5) concludes the proof for both parts.

\section{Corollary 5.4 (H-development property)}

Let $M, N$ be terms such that $M \triangleright N$. Then $\underset{h}{\mathrm{D}} \mathrm{\triangleright}$.

\section{Standardisation}

The part of the standardisation proof following the proof of the h-development property coincides in structure with the proof given in [2].

First we will prove that we can get, for any reduction involving head steps that follows an internal development, another reduction in which the head steps are at the beginning. The name given to the Lemma 6.1 was taken from [2].

This proof needs again to consider explicitly the relations relative to patterns, for similar reasons to those described when introducing h-development in section 3

\section{Lemma 6.1 (Postponement)}

(i) if $M \stackrel{\text { int }}{\triangleright} N \underset{h}{\rightarrow} R$ then there exists some term $N^{\prime}$ such that $M \underset{h}{\rightarrow} N^{\prime} \triangleright R$

(ii) for any pattern $p$, if $M_{\triangleright_{p}}^{i n t} \underset{p}{\underset{p}{\rightsquigarrow}}$ then there exists some term $N_{p}^{\prime}$ such that $\underset{p}{M} N_{p}^{\prime} \triangleright R$ 
Proof For $(i)$, if the rule used in $M \stackrel{i n t}{\triangleright} N$ is IRefl, then the result is immediate taking $N^{\prime}=R$. Therefore, in the following we will ignore this case.

We prove ( $i$ ) and (ii) by simultaneous induction on $M$ taking into account the previous observation.

variable in this case it must be $N=M$ for both (i) and (ii) and neither $M \underset{h}{\rightarrow} R$ nor $M \underset{p}{\rightsquigarrow} R$ for any $p, R$.

abstraction in this case $N$ must also be an abstraction for both $(i)$ and (ii) and neither $N \underset{h}{\rightarrow} R \underset{p}{\operatorname{nor}} \underset{p}{\rightsquigarrow} R$ for any $p, R$.

application in this case $M=M_{1} M_{2}$

We prove (i) first, analysing the possible forms of $M_{1}$

- Assume $M_{1}$ is not an abstraction

In this case IApp1 applies, so we know $N=N_{1} N_{2}, M_{1} \triangleright$ int $N_{1}$, and $M_{2} \triangleright N_{2}$.

Since $M_{1} \stackrel{i n t}{\triangleright} N_{1}, N_{1}$ is not an abstraction, then the only applicable rule for $N \underset{h}{\rightarrow}$ is HApp1, hence $R=R_{1} N_{2}$ and $N_{1} \underset{h}{\rightarrow} R_{1}$.

Now we use the IH on $M_{1} \stackrel{i n t}{\triangleright} N_{1} \underset{h}{\rightarrow} R_{1}$ to get $M_{1} \underset{h}{\rightarrow} N_{1}^{\prime} \triangleright R_{1}$, then we obtain $M=M_{1} M_{2} \underset{h}{\rightarrow}$ $N_{1}^{\prime} M_{2}$ by HApp1.

Finally we apply DApp to $N_{1}^{\prime} \triangleright R_{1}$ and $M_{2} \triangleright N_{2}$ to get $N_{1}^{\prime} M_{2} \triangleright R_{1} N_{2}=R$, which concludes the proof for this case.

- Now assume $M_{1}=\lambda p \cdot M_{12}$ and $p \nless M_{2}$

Since $M=\left(\lambda p . M_{12}\right) M_{2} \stackrel{i n t}{\triangleright} N$, the only rule that applies is IApp2, then $N=\left(\lambda p \cdot N_{12}\right) N_{2}$, $M_{12} \triangleright N_{12}$, and $M_{2} \stackrel{i n t}{p}_{p} N_{2}$. By L. 4.3 we obtain $p \nless N_{2}$, so the only applicable rule in $N=$ $\left(\lambda p \cdot N_{12}\right) N_{2} \underset{h}{\rightarrow} R$ is HPat, then $R=\left(\lambda p \cdot N_{12}\right) R_{2}$ and $N_{2} \underset{p}{\rightsquigarrow} R_{2}$.

Now we use the IH (ii) on $M_{2} \underset{p}{\stackrel{i n t}{\triangleright_{p}}} N_{2} \underset{p}{\rightsquigarrow} R_{2}$, to get $M_{2} \underset{p}{\rightsquigarrow} N_{2}^{\prime} \triangleright R_{2}$.

We obtain $M=\left(\lambda p \cdot M_{12}\right) M_{2} \rightarrow \underset{h}{\rightarrow}\left(\lambda p \cdot M_{12}\right) N_{2}^{\prime}$ by HPat, then we get $\left(\lambda p \cdot M_{12}\right) \triangleright\left(\lambda p \cdot N_{12}\right)$ by DAbs on $M_{12} \triangleright N_{12}$, finally we apply DApp to the previous result and $N_{2}^{\prime} \triangleright R_{2}$ to obtain $\left(\lambda p . M_{12}\right) N_{2}^{\prime} \triangleright\left(\lambda p . N_{12}\right) R_{2}=R$ which concludes the proof for this case.

- Finally, assume $M_{1}=\lambda p \cdot M_{12}$ and $p \ll^{v} M_{2}$

Again, the only rule that applies in $M=\left(\lambda p \cdot M_{12}\right) M_{2} \stackrel{i n t}{\triangleright} N$ is IApp2, then $N=\left(\lambda p \cdot N_{12}\right) N_{2}$, $M_{12} \triangleright N_{12}$, and $M_{2} \triangleright_{p}^{i n t} N_{2}$. Now, by L. 4.2 we obtain $p \ll^{\theta} N_{2}$ for some substitution $\theta$ such that $v \triangleright \theta$, then the applied rule in $N \underset{h}{\rightarrow} R$ is HBeta (the case HPat being excluded by L.4.1), hence $R=\theta N_{12}$

It is clear that $M \underset{h}{\rightarrow} v M_{12}$. By L. 4.6 we obtain $v M_{12} \triangleright \theta N_{12}=R$, which concludes the proof for this case.

For (ii) we proceed by a case analysis of $p$

If $p \in \operatorname{Var}$ then there is no $R$ such that $\underset{p}{\underset{p}{\rightsquigarrow}} R$ for any term $N$.

If $p \ll M$ then by L.4.2 $p \ll N$, and therefore by L. 4.1 there can be no $R$ such that $\underset{p}{N} \underset{p}{ }$. 


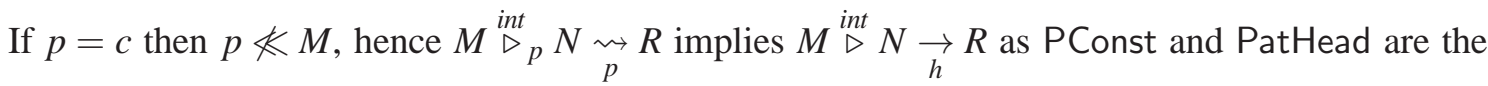
only possibilities for this case respectively. We use part (i) to obtain $M \underset{h}{\rightarrow} N^{\prime} \triangleright R$, and $\underset{p}{\stackrel{p}{~}} N^{\prime}$ by PatHead which concludes the proof for this case.

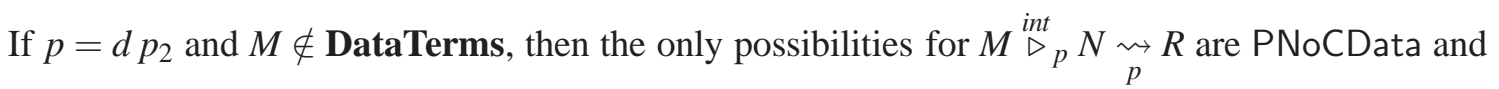
PatHead respectively, then $M \stackrel{i n t}{\triangleright} N \underset{h}{\rightarrow} R$. We use part (i) to obtain $M \underset{h}{\rightarrow} N^{\prime} \triangleright R$, and $\underset{p}{\underset{p}{m}} N^{\prime}$ by PatHead which concludes the proof for this case.

Now assume $p=d p_{2}, M \in$ DataTerms, and $p \nless M$. We must analyse three possibilities

- $d \nless M_{1}$.

In this case only PCDataNo1 applies for $M \stackrel{\text { int }}{\triangleright} p, N$, therefore $N=N_{1} N_{2}$ with $M_{1} \stackrel{i n t}{\triangleright{ }_{d}} N_{1}$ and $M_{2} \triangleright N_{2}$. By L. 4.3 we know $d \nless N_{1}$ and moreover $N_{1}$ is a data term (as can be seen by L. 3.2) thus not having head redexes, so the only possible rule for $\underset{p}{\sim} R$ is Pat1, then $R=R_{1} N_{2}$ with $N_{1} \underset{d}{d} R_{1}$.

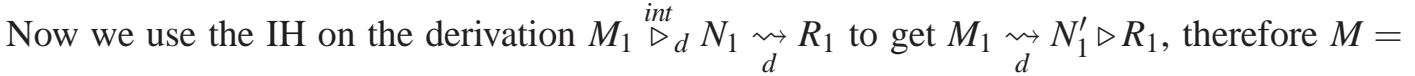
$M_{1} M_{2} \underset{p}{\rightsquigarrow} N_{1}^{\prime} M_{2}$ by Pat1.

Moreover as $N_{1}^{\prime} \triangleright R_{1}$ and $M_{2} \triangleright N_{2}$ hence $N_{1}^{\prime} M_{2} \triangleright R_{1} N_{2}=R$, which concludes the proof for this case.

- $d \ll M_{1}$ and $p_{2} \nless M_{2}$.

In this case only PCDataNo2 applies for $M \stackrel{i n t}{\triangleright}_{p} N$, therefore $N=N_{1} N_{2}$ with $M_{1} \triangleright N_{1}$ and $M_{2} \triangleright_{p_{2}} N_{2}$. By L.4.2 and L. 4.3 respectively, we obtain both $d \ll N_{1}$ and $p_{2} \ll N_{2}$. Moreover $N$ is a data term (as can be seen by L. 3.2) thus not having head redexes. Hence the only possibility for $\underset{p}{\underset{p}{\rightsquigarrow}} R$ is Pat2, then $R=N_{1} R_{2}$ with $N_{2} \underset{p_{2}}{\rightsquigarrow} R_{2}$

We now use the IH on $M_{2}{\stackrel{i n t}{p_{2}}}_{p_{2}} N_{p_{2}} \rightsquigarrow R_{2}$ to get $M_{2} \underset{p_{2}}{\rightsquigarrow} N_{2}^{\prime} \triangleright R_{2}$, and by Pat2 $M=M_{1} M_{2} \underset{p}{\rightsquigarrow} M_{1} N_{2}^{\prime}$ We also use DApp on $M_{1} \triangleright N_{1}$ and $N_{2}^{\prime} \triangleright R_{2}$ to get $M_{1} N_{2}^{\prime} \triangleright N_{1} R_{2}=R$, which concludes the proof for this case.

- $d \ll M_{1}, p_{2} \ll M_{2}$ and $d p_{2} \ll M_{1} M_{2}$.

$d \ll M_{1}$ implies (L 2.5:(b) $) M_{1} \in$ DataTerms so that from $M=M_{1} M_{2} \stackrel{i n t}{\triangleright}{ }_{p} N$ we can only have $N=N_{1} N_{2}$ with $M_{1} \triangleright N_{1}$ and $M_{2} \triangleright N_{2}$. L.4.2 gives $d \ll N_{1}$ and $p_{2} \ll N_{2}$. L. 3.2. (b) gives $N \in$ DataTerms. To show $N \rightsquigarrow R$ we have three possibilities: PatHead is not possible since $N \in$ DataTerms (c.f. L 2.8 (a) $)$ ), Pat1 is not possible since $d \ll M_{1}$ (c.f. L4.1), Pat2 is not possible since $p_{2} \ll N_{2}$ (c.f. L 4.1).

\section{Corollary 6.2}

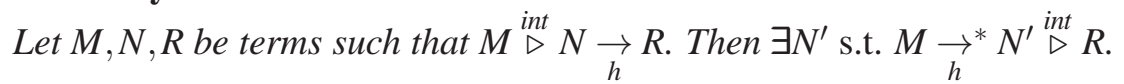

Proof Immediate by L. 6.1 and Corollary 5.4 
Now we generalize the h-development concept to a sequence of developments. The name given to Lemma 6.3 was taken from [2].

\section{Lemma 6.3 (Bifurcation)}

Let $M, N$ be terms such that $M \triangleright^{*} N$. Then $M \vec{h}^{*} R \triangleright^{\text {int }}$. $N$ for some term $R$.

Proof Induction on the length of $M \triangleright^{*} N$. If $M=N$ the result holds trivially.

Assume $M \triangleright Q \triangleright^{*} N$. By C. 5.4 and IH respectively, we obtain $M \vec{h}^{*} S \stackrel{i n t}{\triangleright} Q$ and $Q \underset{h}{*} T \stackrel{i n t}{\triangleright^{*}} N$ for some terms $S$ and $T$. Now we use Corollary 6.2 (many times) on $S \stackrel{\text { int }}{\triangleright} Q \vec{h}^{*} T$ to get $S \underset{h}{*} R \stackrel{\text { int }}{\triangleright} T$.

Therefore $M \underset{h}{\rightarrow} S \underset{h}{*} R \stackrel{i n t}{\triangleright} T \triangleright^{i n t} N$ as we desired.

Using the previous results, the standardisation theorem admits a very simple proof.

Definition 6.4 (Standard reduction sequence) The standard reduction sequences are the sequences of terms $M_{1} ; \ldots ; M_{n}$ which can be generated using the following rules.

$$
\begin{gathered}
\frac{M_{2} ; \ldots ; M_{k} \quad M_{1} \underset{h}{\rightarrow} M_{2}}{M_{1} ; \ldots ; M_{k}} \text { StdHead } \frac{M_{1} ; \ldots ; M_{k}}{\left(\lambda p \cdot M_{1}\right) ; \ldots ;\left(\lambda p \cdot M_{k}\right)} \text { StdAbs } \\
\frac{M_{1} ; \ldots ; M_{j} \quad N_{1} ; \ldots ; N_{k}}{\left(M_{1} N_{1}\right) ; \ldots\left(M_{j} N_{1}\right) ;\left(M_{j} N_{2}\right) ; \ldots ;\left(M_{j} N_{k}\right)} \text { StdApp }-\frac{x}{x} \text { StdVar }
\end{gathered}
$$

\section{Theorem 6.5 (Standardisation)}

Let $M, N$ be terms such that $M \triangleright^{*} N$. Then there exists a standard reduction sequence $M ; \ldots ; N$.

Proof By L. 6.3 we have $M \vec{h}^{*} R \triangleright^{\text {int }} N$; we observe that it is enough to obtain a standard reduction sequence $R ; \ldots ; N$, because we subsequently apply StdHead many times.

Now we proceed by induction on $N$

- $N \in \operatorname{Var}$; in this case $R=N$ and we are done.

- $N=\lambda p \cdot N_{1}$; in this case $R=\lambda p \cdot R_{1}$ and $R_{1} \triangleright^{*} N_{1}$. By IH we obtain a standard reduction sequence $R_{1} ; \ldots ; N_{1}$, then by StdAbs so is $R=\lambda p . R_{1} ; \ldots ; \lambda p . N_{1}=N$.

- $N=N_{1} N_{2}$, so $R=R_{1} R_{2}$ and $N_{i} \triangleright^{*} R_{i}$. We use the IH on both reductions to get two standard reduction sequences $N_{i} ; \ldots ; R_{i}$, then we join them using StdApp.

\section{Conclusion and further work}

We have presented an elegant proof of the Standardisation Theorem for constructor-based pattern calculi.

We aim to generalize both the concept of standard reduction and the structure of the Standardisation Theorem proof presented here to a large class of pattern calculi, including both open and closed variants as the Pure Pattern Calculus [7]. It would be interesting to have sufficient conditions for a pattern calculus 
to enjoy the standardisation property. This will be close in spirit with [8] where an abstract confluence proof for pattern calculi is developed.

The kind of calculi we want to deal with imposes challenges that are currently not handled in the present contribution, such as open patterns, reducible (dynamic) patterns, and the possibility of having fail as a decided result of matching. Furthermore, the possibility of decided fail combined with compound patterns leads to the convenience of studying forms of inherently parallel standard reduction strategies.

The abstract axiomatic Standardisation Theorem developed in [5] could be useful for our purpose. However, while the axioms of the abstract formulation of standardisation are assumed to hold in the proof of the standardisation result, they need to be defined and verified for each language to be standardised. This could be nontrivial, as in the case of TRS [6, 15], where a meta-level matching operation is involved in the definition of the rewriting framework. We leave this topic as further work.

\section{References}

[1] H.P. Barendregt (1984): The Lambda Calculus: Its Syntax and Semantics. Elsevier, Amsterdam.

[2] K. Crary (2009): A Simple Proof of Call-by-Value Standardization. Technical Report CMU-CS-09-137, Carnegie-Mellon University.

[3] H.B. Curry \& R. Feys (1958): Combinatory Logic. North-Holland Publishing Company, Amsterdam.

[4] J.-Y. Girard (1987): Linear Logic. Theoretical Computer Science 50(1), pp. 1-101.

[5] G. Gonthier, J.-J. Lévy \& P.-A. Melliès (1992): An abstract standardisation theorem. In: Proceedings, Seventh Annual IEEE Symposium on Logic in Computer Science, 22-25 June 1992, Santa Cruz, California, USA, IEEE Computer Society, pp. 72-81.

[6] G. Huet \& J.-J. Lévy (1991): Computations in orthogonal rewriting systems. In: Jean-Louis Lassez \& Gordon Plotkin, editors: Computational Logic, Essays in Honor of Alan Robinson, MIT Press, pp. 394-443.

[7] C.B. Jay \& D. Kesner (2006): Pure Pattern Calculus. In: Peter Sestoft, editor: European Symposium on Programming, number 3924 in LNCS, Springer-Verlag, pp. 100-114.

[8] C.B. Jay \& D. Kesner (2009): First-class patterns. Journal of Functional Programming 19(2), pp. $191-225$.

[9] Ryo Kashima (2000): A Proof of the Standardization Theorem in $\lambda$-Calculus. Research Reports on Mathematical and Computing Sciences C-145, Tokyo Institute of Technology.

[10] J.W. Klop, V. van Oostrom \& R.C. de Vrijer (2008): Lambda calculus with patterns. Theoretical Computer Science 398(1-3), pp. 16-31.

[11] Paul-André Melliès (1996): Description Abstraite des Systèmes de Réécriture. Ph.D. thesis, Université Paris VII.

[12] Luca Paolini \& Simona Ronchi Della Rocca (2004): Parametric parameter passing Lambda-calculus. Information and Computation 189(1), pp. 87-106.

[13] G. Plotkin (1975): Call-by-name, call-by-value and the Lambda-calculus. Theoretical Computer Science $1(2)$, pp. 125-159.

[14] M. Takahashi (1995): Parallel reductions in lambda-calculus. Information and Computation 118(1), pp. $120-127$.

[15] Terese (2003): Term Rewriting Systems, Cambridge Tracts in Theoretical Computer Science 55. Cambridge University Press. 